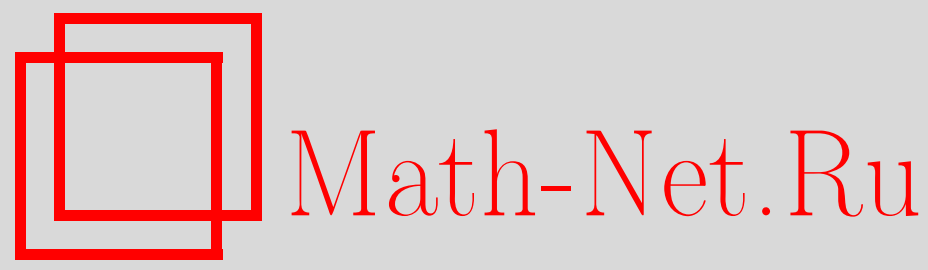

В. Ю. Новокшенов, Динамика уиземовской зоны вещественного решения уравнения синус-Гордон с конечнозонными граничными условиями, Функи. анализ и его прил., 1996, том 30, выпуск 4, 31-44

DOI: https://doi.org/10.4213/faa548

Использование Общероссийского математического портала MathNet.Ru подразумевает, что вы прочитали и согласны с пользовательским соглашением http://www . mathnet.ru/rus/agreement

Параметры загрузки:

IP : 35.174 .16 .151

26 апреля 2023 г., 16:29:30

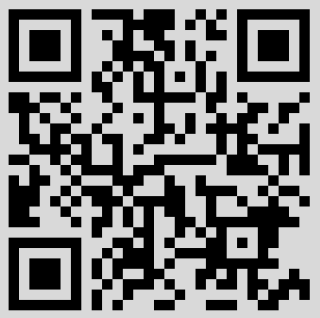




\title{
Динамика уиземовской зоны вещественного решения уравнения синус-Гордон с конечнозонными граничными условиями
}

\author{
(c) $1996 . \quad$ В. Ю. Новокшенов
}

\section{Введение}

Рассмотрим задачу Коши для уравнения синус-Гордон

$$
\begin{gathered}
u_{t t}-u_{x x}+\sin u=0, \\
u(x, 0)=u_{0}(x), \quad u_{t}(x, 0)=u_{1}(x),
\end{gathered}
$$

где начальные данные $u_{0}, u_{1} \in C^{\infty}(\mathbb{R})$ достаточно быстро выходят на конечнозонные граничные функции

$$
\begin{aligned}
& u_{0}(x) \rightarrow \begin{cases}\left.v_{n}\left(\theta_{1}, \ldots, \theta_{n}\right)\right|_{t=0}, & x \rightarrow-\infty, \\
\left.v_{m}\left(\tilde{\theta}_{1}, \ldots, \tilde{\theta}_{m}\right)\right|_{t=0}, & x \rightarrow+\infty,\end{cases} \\
& u_{1}(x) \rightarrow \begin{cases}\left.\partial_{t} v_{n}\left(\theta_{1}, \ldots, \theta_{n}\right)\right|_{t=0}, & x \rightarrow-\infty, \\
\left.\partial_{t} v_{m}\left(\tilde{\theta}_{1}, \ldots, \tilde{\theta}_{m}\right)\right|_{t=0}, & x \rightarrow+\infty,\end{cases} \\
& \theta_{i}=x U_{i}+t V_{i}+W_{i}, \quad i=1, \ldots, n, \\
& \tilde{\theta}_{j}=x \widetilde{U}_{j}+t \widetilde{V}_{j}+\widetilde{W}_{j}, \quad j=1, \ldots, m .
\end{aligned}
$$

Здесь $v_{n}, v_{m}$ суть $2 \pi$-периодические функции по соответствующим аргументам $\theta_{i}, \tilde{\theta}_{j}$, а «обмоточные» векторы $U=\left(U_{1}, \ldots, U_{n}\right), V=\left(V_{1}, \ldots, V_{n}\right)$ выбираются по известным формулам метода конечнозонного интегрирования (см. ниже $\S 1)$ и определяются в конечном счете расположением точек ветвления римановых поверхностей $\Gamma_{n}, \Gamma_{m}$ конечнозонного спектра функций $v_{n}, v_{m}$.

Задача состоит в исследовании асимптотического поведения решения при больших временах. Она относится к классу «задач о ступеньке» для интегрируемых эволюционных уравнений $[1$, гл. 4]. Их теория достаточно хорошо разработана для так называемого случая устойчивых граничных условий (2), когда все точки ветвления вещественны. В этом случае для уравнения (1), также как и в аналогичных задачах с вещественными конечнозонными граничными условиями для уравнений КдФ и НШ (см. [1-7]), главный член асимптотики представляет собой модулированное конечнозонное решение

$$
u=u_{N}\left(\theta_{1}, \ldots, \theta_{N} \mid \xi\right)+o(1), \quad t \rightarrow \infty,
$$

* Работа выполнена при финансовой поддержке Российского фонда фундаментальных исследований (проект №93-01-165), а также Международного научного фонда (грант RK2300). 
где $\theta_{i}=x U_{i}(\xi)+t V_{i}(\xi)+W_{i}(\xi), N=N(\xi), N \in \mathbb{Z}, \xi=x / t$ «медленная» пространственная переменная. Обмоточные векторы $U, V$ и функция $u$ строятся по стандартным формулам типа формул Итса-Матвеева по «подвижной» (зависящей от $\xi$ ) римановой поверхности $\Gamma(\xi)$. В свою очередь, динамика по $\xi$ римановой поверхности (ее точек ветвления) определяется уравнениями Уизема

$$
\left(\xi-S_{j}\left(\lambda_{1}, \ldots, \lambda_{2 N}\right)\right) \frac{d \lambda_{j}}{d \xi}=0, \quad j=1, \ldots, 2 N,
$$

где групповые скорости $S_{j}$ явным образом могут быть выражены в терминах гиперэллиптических интегралов по $a$-циклам поверхности (см. ниже $(1.11),(2.10))$. Подобно случаям КдФ и НШ, здесь все функции $S_{i}$ вещественны и удовлетворяют условиям монотонности $[3-5,7]$

$1^{\circ} . \partial S_{j} / \partial \lambda_{j}<0, j=1, \ldots, 2 N$.

$2^{\circ} . S_{j} \neq S_{i}$ при $\lambda_{j} \neq \lambda_{i}$.

Эти условия обеспечивают существование и единственность кусочно-непрерывного решения системы (6) с граничными условиями [3]

$$
\Gamma(\xi) \rightarrow \begin{cases}\Gamma_{n}, & \xi \rightarrow-\infty \\ \Gamma_{m}, & \xi \rightarrow+\infty\end{cases}
$$

$\mathrm{B}$ действительности граничные значения функций $\lambda_{i}=\lambda_{i}(\xi)$ достигаются при конечных значениях $\xi$. Таким образом, в случае вещественных гиперэллиптических поверхностей $\Gamma_{n}, \Gamma_{m}$, задающих граничные условия (3), (4), главный член (5) асимптотики решения задачи Коши (1), (2) при больших временах полностью определяется динамикой поверхности $\Gamma(\xi)$ - решением системы (6) с граничными условиями (7). Указанная ситуация имеет место и в более общих задачах о пространственно-временных модуляциях волновых пакетов в солитонных уравнениях [14-21]. Так, если $u=u_{k}\left(\theta_{1}, \ldots, \theta_{k}\right)$ есть $k$-зонное решение уравнения (1), то возмушенное по медленным переменным решение должно иметь вид

$$
u=u_{k}\left(\theta_{1}(X, T), \ldots, \theta_{k}(X, T)\right)+o(1), \quad \varepsilon \rightarrow 0,|x|,|t|<\text { const } \cdot \varepsilon,
$$

где фазы $\theta_{i}=x U_{i}(X, T)+t V_{i}(X, T)+W_{i}(X, T)$ определяются по римановой поверхности $\Gamma=\Gamma(X, T)$, удовлетворяющей гиперболической системе Уизема

$$
\frac{\partial \lambda_{j}}{\partial T}-S_{j}\left(\lambda_{1}, \ldots, \lambda_{2 N}\right) \frac{\partial \lambda_{j}}{\partial X}=0, \quad j=1, \ldots, 2 N,
$$

где $S_{j}$ совпадают с групповыми скоростями, фигурирующими в системе $(6)$ (см. [2]). Нетрудно видеть, что (6) представляет собой автомодельную редукцию системы (8). В ряде случаев при специальных граничных условиях (7) (типа центрированных волн разрежения) можно доказать, что асимптотически при $T \rightarrow \infty$ решение (8) при условиях монотонности на $S_{j}$ сходится к решению системы (6) (см. [3-7]).

Все сказанное выше относится к вещественным поверхностям $\Gamma$, определенным выбором вещественных точек ветвления $\lambda_{j}$. Модулированное конечнозонное решение (5) в этом случае по терминологии [2] представляет собой «kink-train» - устойчивое по малым возмущениям асимптотическое решение. Этот факт обусловлен в конечном счете вещественностью и строгой разделенностью групповых 
скоростей в уравнениях (6), (8). Качественно новая ситуация возникает при замене вещественных поверхностей $\Gamma_{n}, \Gamma_{m}$ для граничных условий (3), (4) на самосопряженные поверхности. При подходящем выборе базиса циклов и абелевых дифференциалов гарантируется вещественность граничных функций (см. [2, 10]) и тем самым вещественность решения задачи (1), (2). Однако попытка использовать модулированный конечнозонный анзатц (5) для главного члена асимптотики при больших временах приводит к системе Уизема (6) с комплексными групповыми скоростями. Вещественная редукция обеспечивает вещественность анзатца (5), однако этот анзатц («breather-train» по терминологии [2]) будет неустойчив относительно малых возмущений. Тем не менее мы будем предполагать в качестве гипотезы (пока не доказанной), что асимптотика решений задачи (1), (2) при $t \rightarrow \infty$ имеет структуру модулированного конечнозонного решения $(5)$, параметры которого следует определить из системы (6) с граничными условиями (7).

В пользу этой гипотезы свидетельствуют накопленные в последнее время факты о поведении неустойчивых решений солитонных уравнений [8, 16-23]. Дело в том, что линейная теория возмущений не дает правильных результатов в неустойчивом случае, поскольку в точно интегрируемых системах большое возмущение не разрушает решений, а лишь переводит их в новое «метастабильное состояние». Классической иллюстрацией здесь служит математический маятник $\ddot{u}=\sin u$ в окрестности неустойчивого положения равновесия $u=0$. В интегрируемой системе (1) существует большой запас условно-периодических решений, служащих, как отмечено в [8], аттракторами для (условно-периодических) возмущений таких решений. Механизм их эволюции по медленным переменным является, по-видимому, универсальным как в устойчивом, так и в неустойчивом случае и описывается системой Уизема (5) (см. [16-19, 23]).

Ясно, что в неустойчивом случае вопрос о разрешимости системы Уизема становится нетривиальным ввиду появления равенств вида

$$
\xi=S_{j}\left(\lambda_{1}, \ldots, \lambda_{2 N}\right), \quad j=1, \ldots, 2 N,
$$

где $\xi$ вещественно, а $S_{j}$ комплексны. В этом случае мнимые и вещественные части системы (9) определяют «кинематику» и «динамику» точек ветвления, а именно уравнения $\operatorname{Im} S_{j}=0$ определяют траектории $\lambda_{k}$ в комплексной плоскости, в то время как уравнения $\operatorname{Re} S_{j}=\xi$ задают закон движения точек по этим траекториям. Ввиду сильной нелинейности функций $S_{j}$ траектории точек ветвления могут выглядеть весьма нетривиально, а доказательство оценок вида $1^{\circ}, 2^{\circ}$ значительно сложнее, чем в вещественном случае. Основным результатом работы является доказательство разрешимости системы Уизема (8) при $N=2$ для произвольных несовпадающих однозонных граничных условий (7) с комплексно-сопряженными точками ветвления.

\section{$\S 1$. Конструкция уравнений Уизема}

Приведем здесь кратко основные формулы для конечнозонных решений уравнения синус-Гордон (1) и дадим эвристический вывод уравнений Уизема (6). 
Вешественное $N$-зонное решение уравнения (1) строится по заданной гиперэллиптической поверхности $\Gamma$ вида $\zeta^{2}=\left(\lambda-\lambda_{1}\right) \cdots\left(\lambda-\lambda_{2 N}\right)$, подчиненной антиголоморфной инволющии $\tau:(\lambda, \zeta) \mapsto(\bar{\lambda},-\bar{\zeta})$. Ограничимся рассмотрением поверхностей с условиями $\lambda_{0}=0, \lambda_{j+N}=\bar{\lambda}_{j}, j=1, \ldots, N$. Определим базис циклов, как показано на рис. 1.

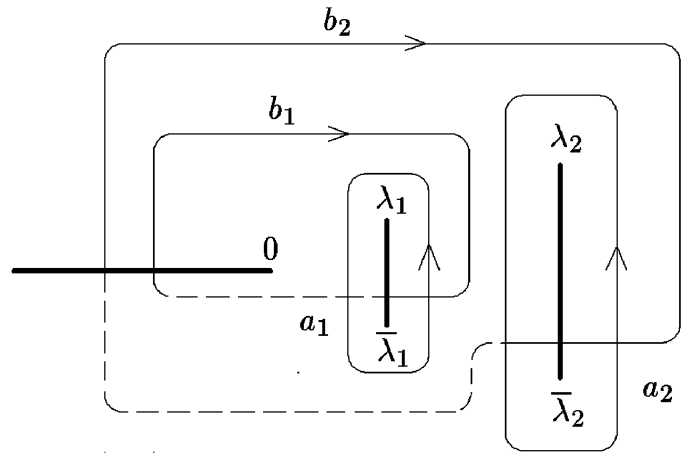

Рис. 1. Базис циклов на поверхности $Г$. Пунктиром обозначен путь на нижнем листе.

Выберем базис $\omega_{j}, j=1, \ldots, N$, голоморфных абелевых дифференциалов на $\Gamma$ с нормировкой

$$
\int_{a_{k}} \omega_{j}=\delta_{i j}, \quad k, j=1, \ldots, N
$$

Определим пару абелевых дифференциалов второго рода $\Omega_{ \pm 1}$, так что $\Omega_{+1}$ аналитичен на $\Gamma$ всюду, кроме $\lambda=\infty$, а дифференциал $\Omega_{-1}$ аналитичен всюду, кроме $\lambda=0$, причем в окрестности этих точек справедливо представление $\Omega_{ \pm 1}=\xi^{-2} d \xi+O(1)$, где $\xi-$ локальный параметр. Дифференциалы однозначно фиксируются нормировкой

$$
\int_{a_{k}} \Omega_{ \pm 1}=0, \quad k=1, \ldots, N
$$

Указанный выбор абелевых дифференциалов обеспечивает существование вешественного неособого решения уравнения (1), задаваемого формулой [9]

$$
u_{N}(x, t)=2 i \log \frac{\theta(x U+t V+1 / 2+D)}{\theta(x U+t V+D)},
$$

где $\theta$ - тета-функция Римана на $\Gamma$ с матрицей периодов $B_{i j}=\int_{b_{j}} \omega_{i}, D$ чисто мнимый постоянный вектор, а компоненты обмоточных векторов $U=$ $-4 i\left(U_{1}, \ldots, U_{N}\right), V=-4 i\left(V_{1}, \ldots, V_{N}\right)$ совпадают с интегралами по $b$-циклам от дифференциалов $\Omega_{+}$и $\Omega_{-}$соответственно, $\Omega_{ \pm}=\Omega_{+1} \pm \Omega_{-1} / 16$.

Вектор-функция $\Psi(P, x, t)=\left(\Psi_{-}, \Psi_{+}\right)^{T}, P=(\lambda, \zeta)$, удовлетворяющая уравнениям $L-A$-пары для уравнения синус-Гордон $(1)$, является в случае $u=$ 
$u_{N}$ аналитической на поверхности $\Gamma$ и имеет вид [11]

$$
\Psi_{ \pm}=\frac{\theta(\mathscr{A}(P)+x U+t V+D+(1 \pm 1) / 4) \theta(D)}{\theta(\mathscr{A}(P)+D) \theta(x U+t V+D+(1 \pm 1) / 4)} \exp \left[-4 i \int_{\infty}^{P}\left(x \Omega_{-}+t \Omega_{+}\right)\right],
$$

где $\mathscr{A}(P)=\left(\int_{\infty}^{P} \omega_{1}, \ldots, \int_{\infty}^{P} \omega_{N}\right)$ - отображение Абеля.

Опираясь на приведенные выше формулы для точного $N$-зонного решения уравнения (1), установим динамику параметров, задающих «модуляцию» этого решения по медленной переменной $\xi=x / t$. Будем предполагать [7], что модулированное $N$-зонное решение

$$
\tilde{u}=u_{N}(x, t \mid \xi)+o(1), \quad t \rightarrow \infty
$$

определено формулой (1.2), где все величины заданы на «подвижной» римановой поверхности $\Gamma=\Gamma(\xi)$, т. е. поверхности, имеющей точки ветвления $\lambda_{j}=\lambda_{j}(\xi)$. Тогда решение (1.4) удовлетворяет уравнению (1) с точностью $о(1)$ на интервале длины $O(t)$ только в том случае, когда в соответствующих уравнениях $L$-A-пары для модулированной функции $\widetilde{\Psi}=\widetilde{\Psi}(P, x, t \mid \xi)$ вида $(1.3)$ нет секулярных (порядка $\left.O\left(t^{-1}\right)\right)$ членов.

Для зануления секулярных членов достаточно потребовать, очевидно, чтобы $\widetilde{\Psi}_{\xi}=0$, а это, в свою очередь, в силу (1.3) влечет за собой

$$
\int_{\infty}^{P}\left(\xi \frac{\partial \Omega_{-}}{\partial \xi}+\frac{\partial \Omega_{+}}{\partial \xi}\right)=0, \quad P \in \Gamma .
$$

Поскольку в окрестности точек ветвления дифференциалы имеют вид

$$
\Omega_{ \pm}=\operatorname{const} \cdot \frac{d \lambda}{\sqrt{\lambda-\lambda_{j}(\xi)}}+O\left(\sqrt{\lambda-\lambda_{j}(\xi)}\right)
$$

равенство (1.5) при $\lambda \rightarrow \lambda_{j}$ эквивалентно $\left(\xi-S_{j}\right) \lambda_{j}^{\prime}(\xi)=0$, где

$$
S_{j}=S\left(\lambda_{j} \mid \lambda_{1}, \lambda_{2}, \ldots, \lambda_{N}\right)=\left.\left(-\Omega_{+} / \Omega_{-}\right)\right|_{\lambda=\lambda_{j}}, \quad S_{j+N}=\bar{S}_{j}, \quad j=1, \ldots, N .
$$

Полученные уравнения доставляют необходимые условия существования $N$-зонного решения, модулированного параметром $\xi=x / t$. Если учитывать в них вещественную редукцию $\lambda_{j+N}=\bar{\lambda}_{j}$ поверхности $\Gamma$, то система Уизема приходит к виду

$$
\left(\xi-S\left(\lambda_{k} \mid \lambda_{1}, \lambda_{2}, \ldots, \lambda_{N}\right)\right) \lambda_{k}^{\prime}=0, \quad k=1, \ldots, N
$$

\section{§2. Свойства групповых скоростей}

Ключевую роль в исследовании свойств коэффициентов $S_{j}$ уравнения Уизема (1.6) играет их аналитическое представление в терминах гиперэллиптических интегралов по $a$-циклам поверхности Г. 
Положим $R(\mu)=\sqrt{\mu\left(\mu-\lambda_{1}\right)\left(\mu-\bar{\lambda}_{1}\right) \cdots\left(\mu-\lambda_{N}\right)\left(\mu-\bar{\lambda}_{N}\right)}$ и составим определитель из гиперэллиптических интегралов

$$
\left|p_{1}(\mu), \ldots, p_{N}(\mu)\right| \stackrel{\text { def }}{=}\left\{\int_{a_{j}} \frac{p_{i}(\mu)}{R(\mu)} d \mu\right\}_{i, j=1, \ldots, N},
$$

где $p_{1}, \ldots, p_{N}$ - рациональные по $\mu$ функции. Условимся также, что в обозначениях (1.6) для $S_{j}=S\left(\lambda_{j} \mid \lambda_{1}, \ldots, \lambda_{N}\right)$ зависимость от $\lambda_{1}, \ldots, \lambda_{N}$ будет опушена, если это не приводит к недоразумениям. Теорема $2.1[2]$. Отношение абелевых дифференииалов $S(\lambda)=-\frac{\Omega_{+}(\lambda)}{\Omega_{-}(\lambda)}$
представимо в виде

$$
S(\lambda)=\frac{\left|\lambda-\mu,(\lambda-\mu) \mu, \ldots,(\lambda-\mu)\left(c \mu^{-1}+\lambda \mu^{N-1}\right)\right|}{\left|\lambda-\mu,(\lambda-\mu) \mu, \ldots,(\lambda-\mu)\left(c \mu^{-1}-\lambda \mu^{N-1}\right)\right|},
$$

где $c=\left|\lambda_{1} \cdots \lambda_{N}\right| / 16$.

ДокАЗАТЕЛЬСТво. По определению дифференциалы имеют вид

$$
\begin{aligned}
& \Omega_{+1}=\frac{\left(\lambda-A_{1}\right) \cdots\left(\lambda-A_{N}\right)}{R(\lambda)} d \lambda, \\
& \Omega_{-1}=\frac{\left|\lambda_{1} \cdots \lambda_{N}\right|\left(\lambda B_{1}-1\right) \cdots\left(\lambda B_{N}-1\right)}{\lambda R(\lambda)} d \lambda,
\end{aligned}
$$

причем постоянные $A_{j}, B_{j}$ фиксируются условиями нормировки по $a$-циклам (1.1). Разрешая получающуюся таким образом систему линейных уравнений, приходим к представлению (2.2) для дифференциалов $\Omega_{ \pm}=\Omega_{+1} \pm \Omega_{-1} / 16$. Подробности вычислений приведены в [2, р. 27-30].

Теорема 2.2. Пусть $\lambda$-одна из точек ветвления; тогда равенство $\operatorname{Im} S(\lambda)=0$ эквивалентно равенству $\left|\Lambda \mu^{-1}, \Lambda, \ldots, \Lambda \mu^{N-2}\right|=0$, где $\Lambda=$ $(\lambda-\mu)(\bar{\lambda}-\mu)$.

ДокАЗАТЕЛЬСТво. Определители, фигурирующие в формуле (2.2), можно представить в виде

$$
\Omega_{ \pm}=(-1)^{N} \bar{\lambda}^{-1}\left|\lambda-\mu, \Lambda, \Lambda \mu, \ldots, \Lambda \mu^{N-3}, \Lambda\left(c \mu^{-1} \pm|\lambda|^{2} \mu^{N-2}\right)\right| ;
$$

тогда в силу вещественности интегралов по $a$-циклам от $\Lambda \mu^{j}$ мнимая часть отношения $S$ принимает вид

$$
\begin{aligned}
2 \operatorname{Im} S(\lambda)= & \frac{\left|\lambda-\mu, \mathscr{M}, \Lambda\left(c \mu^{-1}-|\lambda|^{2} \mu^{N-2}\right)\right|}{\left|\Omega_{-}(\lambda)\right|^{2}}\left|\bar{\lambda}-\mu, \mathscr{M}, \Lambda\left(\bar{c} \mu^{-1}+|\lambda|^{2} \mu^{N-2}\right)\right| \\
& -\frac{\left|\bar{\lambda}-\mu, \mathscr{M}, \Lambda\left(c \mu^{-1}-|\lambda|^{2} \mu^{N-2}\right)\right|}{\left|\Omega_{-}(\lambda)\right|^{2}}\left|\lambda-\mu, \mathscr{M}, \Lambda\left(c \mu^{-1}+|\lambda|^{2} \mu^{N-2}\right)\right| \\
= & \frac{|\lambda-\mu, \mathscr{M}, \bar{\lambda}-\mu|}{\left|\Omega_{-}(\lambda)\right|^{2}}\left|\Lambda\left(c \mu^{-1}-|\lambda|^{2} \mu^{N-2}\right), \mathscr{M}, \Lambda\left(c \mu^{-1}+|\lambda|^{2} \mu^{N-2}\right)\right| \\
= & \frac{\Delta_{1} \Delta_{2}}{\left|\Omega_{-}(\lambda)\right|^{2}} .
\end{aligned}
$$


Здесь прямоугольная $(N-2) \times N$-матрица $\mathscr{M}$ составлена из столбцов $\Lambda, \Lambda \mu$, $\ldots, \Lambda \mu^{N-3}$. Второе равенство в (2.3) есть следствие известного равенства из теории определителей

$$
|a, \mathscr{M}, b| \cdot|c, \mathscr{M}, d|-|c, \mathscr{M}, b| \cdot|a, \mathscr{M}, d|=|a, \mathscr{M}, d| \cdot|b, \mathscr{M}, c|,
$$

где $a, b, c$ и $d-$ произвольные столбцы, а $\mathscr{M}-$ произвольная $(N-2) \times N$ матрица.

Пользуясь далее элементарными свойствами определителей, множители в (2.3) легко преобразовать к виду

$$
\begin{aligned}
& \Delta_{1}=|\lambda-\mu, \mathscr{M}, \bar{\lambda}-\mu|=(\bar{\lambda}-\lambda)\left|1, \mu, \mu^{2}, \ldots, \mu^{N-1}\right|, \\
& \Delta_{2}=\left.\left.\left|\Lambda c \mu^{-1}, \mathscr{M},\right| \lambda\right|^{2} \mu^{N-2} \Lambda|=c| \lambda\right|^{2}\left|\Lambda \mu^{-1}, \Lambda, \ldots, \Lambda \mu^{N-2}\right| .
\end{aligned}
$$

Согласно предложению 1 (см. приложение), $\Delta_{1} \neq 0$; следовательно, при $\lambda \neq 0$ из $\operatorname{Im} S(\lambda)=0$ вытекает $\Delta_{2}=0$. Обратное утверждение следует из того, что в точках ветвления дифференциал $\Omega_{-}$не равен нулю [11]. Теорема доказана.

Лемма 2.1. Пусть $\lambda и \lambda^{*}$-две различнье точки; тогда имеет место представление

$$
S(\lambda)-S\left(\lambda^{*}\right)=c \lambda \lambda^{*} \frac{\left(\lambda-\lambda^{*}\right)\left|1, \mu, \ldots, \mu^{N-1}\right|}{\Omega_{-}(\lambda) \Omega_{-}\left(\lambda^{*}\right)}\left|\Lambda \mu^{-1}, \Lambda, \ldots, \Lambda \mu^{N-2}\right|,
$$

где $\Lambda=(\lambda-\mu)\left(\lambda^{*}-\mu\right)$.

ДокАЗАТЕЛЬСтво. Перепишем определители в формуле (2.2) в виде

$$
\Omega_{ \pm}=(-1)^{N}\left(\lambda^{*}\right)^{-1}\left|\lambda-\mu, \Lambda, \Lambda \mu, \ldots, \Lambda \mu^{N-3}, \Lambda\left(c \mu^{-1} \pm \lambda^{*} \lambda \mu^{n-2}\right)\right|,
$$

где $\Lambda=(\lambda-\mu)\left(\lambda^{*}-\mu\right)$. Тогда для разности справедливо разложение в произведение

$$
\begin{aligned}
S(\lambda)- & S\left(\lambda^{*}\right) \\
= & \frac{\left|\lambda-\mu, \mathscr{M}, \Lambda\left(c \mu^{-1}+\lambda^{*} \lambda \mu^{N-2}\right)\right|}{\Omega_{-}(\lambda) \Omega_{-}\left(\lambda^{*}\right)}\left|\lambda^{*}-\mu, \mathscr{M}, \Lambda\left(c \mu^{-1}-\lambda^{*} \lambda \mu^{N-2}\right)\right| \\
& \quad-\frac{\left|\lambda^{*}-\mu, \mathscr{M}, \Lambda\left(c \mu^{-1}+\lambda^{*} \lambda \mu^{N-2}\right)\right|}{\Omega_{-}(\lambda) \Omega_{-}\left(\lambda^{*}\right)}\left|\lambda-\mu, \mathscr{M}, \Lambda\left(c \mu^{-1}-\lambda^{*} \lambda \mu^{N-2}\right)\right| \\
= & \frac{\left|\lambda-\mu, \mathscr{M}, \lambda^{*}-\mu\right|}{\Omega_{-}(\lambda) \Omega_{-}\left(\lambda^{*}\right)}\left|\Lambda\left(c \mu^{-1}-\lambda^{*} \lambda \mu^{N-2}\right), \mathscr{M}, \Lambda\left(c \mu^{-1}+\lambda^{*} \lambda \mu^{N-2}\right)\right| \\
= & \frac{\Delta_{1} \Delta_{2}}{\Omega_{-}(\lambda) \Omega_{-}\left(\lambda^{*}\right)} .
\end{aligned}
$$

Здесь $\mathscr{M}=\left(\Lambda, \Lambda \mu, \ldots, \Lambda \mu^{N-3}\right)$ и во втором равенстве использовано соотношение (2.4). Применяя далее элементарные преобразования определителей вида $(2.5),(2.6)$, получаем представление (2.7). Лемма доказана.

ТеОрема 2.3. Пусть $\lambda_{j}, \lambda_{k}-$ две различные точки ветвления, такие, что либо $\operatorname{Im} S\left(\lambda_{j}\right)=0$, либо $\operatorname{Im} S\left(\lambda_{j}\right)=0$. Тогда $S\left(\lambda_{j}\right) \neq S\left(\lambda_{k}\right)$.

ДокаЗАТЕЛЬСтво. Составим разность $S\left(\lambda_{j}\right)-S\left(\lambda_{k}\right)$, которая по лемме 2.1 имеет представление (2.7). Поскольку по предложению 1 (см. приложение) нули 
имеет лишь второй определитель в $(2.7)$, а $\Omega_{-}\left(\lambda_{j}\right) \Omega_{-}\left(\lambda_{k}\right) \neq 0, \lambda_{j} \neq \lambda_{k}$, то равенство $S\left(\lambda_{j}\right)=S\left(\lambda_{k}\right)$ имеет место лишь при $\left|\Lambda \mu^{-1}, \Lambda, \ldots, \Lambda \mu^{N-2}\right|=0$. Однако последнее невозможно, если $\operatorname{Im} S\left(\lambda_{j}\right)=0$ или $\operatorname{Im} S\left(\lambda_{k}\right)=0$, по предложению 2 из приложения. Полученное противоречие доказывает теорему.

СлЕдСтвиЕ. Пусть $\operatorname{Im} S\left(\lambda_{j}\right)=0$ в точке ветвления. Тогда $\partial S\left(\lambda_{j}\right) / \partial \lambda_{j}$ $\neq 0$.

ДокАЗАТЕЛЬСТВо. Пусть точка $\lambda$ лежит достаточно близко к $\lambda_{j}$, так что $\Omega_{-}(\lambda) \neq 0$. Тогда формула $(2.7)$ переписывается в виде

$$
\frac{S\left(\lambda_{j}\right)-S(\lambda)}{\lambda_{j}-\lambda}=\frac{c \lambda_{j} \lambda\left|1, \mu, \ldots, \mu^{N-1}\right|}{\Omega_{-}\left(\lambda_{j}\right) \Omega_{-}(\lambda)}\left|\Lambda \mu^{-1}, \Lambda, \ldots, \Lambda \mu^{N-2}\right|,
$$

где $\Lambda=(\lambda-\mu)\left(\lambda_{j}-\mu\right)$. Переходя к пределу при $\lambda \rightarrow \lambda_{j}$, получим

$$
\begin{aligned}
& \left.\frac{\partial S}{\partial \lambda}\left(\lambda \mid \lambda_{1}, \ldots, \lambda_{N}\right)\right|_{\lambda=\lambda_{j}} \\
& \quad=\frac{c \lambda_{j}^{2}\left|1, \mu, \ldots, \mu^{N-1}\right|}{\Omega_{-}^{2}\left(\lambda_{j}\right)}\left|\left(\lambda_{j}-\mu\right)^{2} \mu^{-1},\left(\lambda_{j}-\mu\right)^{2}, \ldots,\left(\lambda_{j}-\mu\right)^{2} \mu^{N-2}\right| .
\end{aligned}
$$

Согласно предложению 1 и следствию предложения 2, правая часть здесь отлична от нуля.

Оценим теперь полную производную $\partial S\left(\lambda_{j} \mid \lambda_{1}, \ldots, \lambda_{j}, \ldots, \lambda_{N}\right) / \partial \lambda_{j}$. Как видно из формулы $(2.2)$, величины $\lambda$ входят в $S_{j}$ только посредством гиперэллиптических интегралов от $\mu^{k}(\mu-\lambda) R^{-1}(\mu) d \mu$; поэтому

$$
\frac{\partial S\left(\lambda_{j} \mid \lambda_{1}, \ldots, \lambda_{j}, \ldots, \lambda_{N}\right)}{\partial \lambda_{j}}=\left.\frac{1}{2} \frac{\partial S\left(\lambda \mid \lambda_{1}, \ldots, \lambda_{N}\right)}{\partial \lambda}\right|_{\lambda=\lambda_{j}} .
$$

Последняя производная отлична от нуля в силу (2.8). Следствие доказано.

\section{§3. Динамика уиземовских зон}

Вернемся теперь к анализу решений системы Уизема (1.6). Условимся называть решением системы (1.6) набор кусочно-гладких функций $\left\{\lambda_{1}(\xi), \ldots, \lambda_{N}(\xi)\right\}$, гладких на конечном множестве интервалов $\left(-\infty, \xi_{1}\right),\left(\xi_{1}, \xi_{2}\right), \ldots,\left(\xi_{l-1}, \xi_{l}\right)$, $\left(\xi_{l}, \infty\right)$ и непрерывных в граничных точках ${ }^{1}$. Граничные точки $\xi_{i}$ будут определяться как «моменты схлопывания» или «рождения» точек ветвления, т.е. $\operatorname{Im} \lambda_{j}(\xi)=0$ или $\lambda_{j}\left(\xi_{i}\right)=\lambda_{k}\left(\xi_{i}\right)$. На каждом интервале будем называть точку $\lambda_{j}$ подвижной, если $\lambda_{j}^{\prime}(\xi) \neq 0$.

ТЕОрема 3.1. В каждый «момент времени» $\xi$ среди решений системь (3.1) подвижной является не более чем одна точка $\lambda_{j}$, причем $\lambda_{j}^{\prime}<\infty$.

ДокаЗАТЕЛЬСтво. Пусть, напротив, при некотором $\xi$ подвижны точки $\lambda_{k}$ и $\lambda_{j}$. Тогда $k$-е и $j$-е уравнения системы (1.6) превращаются в равенства $\xi=$ $S\left(\lambda_{k}\right), \xi=S\left(\lambda_{j}\right), \operatorname{Im} S\left(\lambda_{j}\right)=\operatorname{Im} S\left(\lambda_{k}\right)=0$. Однако в силу последних равенств по теореме 2.3 имеем $S\left(\lambda_{k}\right) \neq S\left(\lambda_{j}\right)$. По следствию теоремы $2.3 \lambda_{j}^{\prime}(\xi)=$ $\left(\partial S / \partial \lambda_{j}\right)^{-1}<\infty$. Полученное противоречие доказывает теорему.

${ }^{1}$ По поводу гладкости решения $u(x, t)$ в граничных точках см. замечание 1 в конце $\S 3$. 
Таким образом, на каждом интервале $\xi_{i}<\xi<\xi_{i+1}$ подвижная точка $\lambda_{j}=$ $\lambda_{j}(\xi)$ определяется как решение системы функциональных уравнений

$$
\begin{aligned}
& \operatorname{Im} S\left(\lambda_{j}(\xi) \mid \lambda_{1}, \ldots, \lambda_{j-1}, \lambda_{j}(\xi), \lambda_{j+1}, \ldots, \lambda_{N}\right)=0, \\
& \operatorname{Re} S\left(\lambda_{j}(\xi) \mid \lambda_{1}, \ldots, \lambda_{j-1}, \lambda_{j}(\xi), \lambda_{j+1}, \ldots, \lambda_{N}\right)=\xi,
\end{aligned}
$$

где точки $\lambda_{k} \neq \lambda_{j}$ фиксированы. Уравнение (3.1) задает кривую в комплексной плоскости $\lambda$, в то время как уравнение (3.2) задает динамику движения точки $\lambda_{j}$ вдоль этой кривой. Рассмотрим сначала свойства решений уравнения (3.1). Будем опускать для краткости индекс $j$, считая $\lambda=\lambda(\xi)$ одной из точек ветвления.

ЛЕмма 3.1. Кривая $\lambda=\lambda(\xi)$, множество решений уравнения (3.1), проходит через остальнье точки ветвления $\lambda_{1}, \ldots, \lambda_{N}$.

ДокАЗАтЕЛьство. Утверждение леммы означает, что $\operatorname{Im} S\left(\lambda_{i} \mid \lambda_{1}, \ldots, \lambda_{N}\right)$ $=0$. По теореме 2.2 достаточно доказать, что

$$
\left|\left(\lambda_{i}-\mu\right)\left(\bar{\lambda}_{i}-\mu\right) \mu^{-1}, \ldots,\left(\lambda_{i}-\mu\right)\left(\bar{\lambda}_{i}-\mu\right) \mu^{N-2}\right|=0 .
$$

По построению $j$-я строка последнего определителя составлена из абелевых интегралов по циклу $a_{j}$. При $\lambda_{i}=\lambda_{j}$ циклы $a_{i}$ и $a_{j}$ совпадают, и определитель обращается в нуль. Лемма доказана.

ЛЕмма 3.2. Кривая $\lambda=\lambda(\xi)$, проходяшая через точку ветвления $\lambda_{i}$, пересекает вещественную ось.

ДокАЗАтЕЛЬСтво. Пусть, напротив, множество решений уравнения (3.1), содержащее точку $\lambda_{i}$, лежит строго в верхней полуплоскости. Функция $S\left(\lambda \mid \lambda_{1}, \ldots\right.$, $\left.\lambda, \ldots, \lambda_{N}\right)$ на этой кривой вещественна и имеет, следовательно, максимум и минимум в некоторых точках $\lambda_{\max }$ и $\lambda_{\min }$. Поскольку функция $S$ конечна в точке ветвления и $S(\infty)=1$, то хотя бы одна из этих точек не совпадает с бесконечностью. Тогда в этой точке $\partial S / \partial \lambda=0$, что противоречит следствию теоремы 2.3. Лемма доказана.

Обращаясь к уравнению $(3.2)$, заметим что производная $\lambda^{\prime}(\xi)=1 / S_{\lambda}$ не равна нулю и конечна по следствию теоремы 2.3. Отсюда вытекает следующее утверждение:

Лемма 3.3. Движение точки $\lambda=\lambda(\xi)$ вдоль кривой $\operatorname{Im} S(\lambda)=0$ происходит монотонно и с конечной скоростью.

Теорема 3.2. Пусть $\lambda=\lambda(\xi)$ - решение уравнений (3.1), (3.2) в некотором интервале $\xi_{\text {нач }}<\xi<\xi_{\text {кон }}$. Тогда это решение единственно.

ДокАЗАтЕльство. Пусть, напротив, существуют два решения $\lambda=\lambda(\xi)$ и $\lambda^{*}=\lambda^{*}(\xi)$, такие, что $\operatorname{Im} S(\lambda(\xi))=0$ и $\operatorname{Im} S\left(\lambda^{*}(\xi)\right)=0$ в силу уравнения $(3.1)$. Тогда из уравнения (3.2) имеем $\xi=\operatorname{Re} S(\lambda(\xi))=\operatorname{Re} S\left(\lambda^{*}(\xi)\right)$, что противоречит теореме 2.3. Теорема доказана.

Лемма 3.4. Пусть $N=2,\left|\lambda_{1}\right|=\left|\lambda_{2}\right|, \operatorname{Im} S\left(\lambda_{1}\right)=0$ и точка $\lambda_{2}$ неподвижна. Тогда траектория подвижной точки $\lambda_{1}=\lambda(\xi)$ лежит на окружности $\left|\lambda_{1}\right|=\left|\lambda_{2}\right|$.

ДокАЗАтЕЛЬСтво. По теореме 2.2 уравнение (3.1) при $N=2$ принимает вид

$$
\left|\left(\lambda_{1}-\mu\right)\left(\bar{\lambda}_{1}-\mu\right) \mu^{-1},\left(\lambda_{1}-\mu\right)\left(\bar{\lambda}_{1}-\mu\right)\right|=0
$$


Предложение 3 (см. приложение) утверждает, что все точки окружности $\left|\lambda_{1}\right|=$ $\left|\lambda_{2}\right|$ удовлетворяют уравнению (3.3). Других решений системы (3.1), (3.2) нет в силу теоремы единственности (теорема 3.2). Лемма доказана.

Докажем теперь существование и единственность уиземовской перестройки в случае однозонных граничных условий $\Gamma_{j}: \zeta^{2}=\lambda\left(\lambda-\lambda_{j}\right)\left(\lambda-\bar{\lambda}_{j}\right), j=1,2$. Будем искать эту перестройку в виде

$$
\widetilde{\Gamma}(\xi)= \begin{cases}\Gamma_{1}, & \xi<\xi_{\text {нач }}, \\ \widetilde{\Gamma}(\xi, 1), & \xi_{\text {нач }}<\xi<\xi_{1}, \\ \Gamma_{12}, & \xi_{1}<\xi<\xi_{2}, \\ \widetilde{\Gamma}(\xi, 2), & \xi_{2}<\xi<\xi_{\text {кон }}, \\ \Gamma_{2}, & \xi>\xi_{\text {кон }},\end{cases}
$$

где $\widetilde{\Gamma}(\xi, j)$ - двухзонные поверхности,

$\widetilde{\Gamma}(\xi, j): \quad \zeta^{2}=\lambda(\lambda-\lambda(\xi))(\lambda-\bar{\lambda}(\xi))\left(\lambda-\lambda_{j}\right)\left(\lambda-\bar{\lambda}_{j}\right), \quad j=1,2, \quad \xi_{\text {нач }}<\xi<\xi_{\text {кон }}$,

так что $\widetilde{\Gamma}\left(\xi_{\text {нач }}, 1\right)=\Gamma_{1}, \widetilde{\Gamma}\left(\xi_{\text {кон }}, 2\right)=\Gamma_{2}, \widetilde{\Gamma}\left(\xi_{1}, 1\right)=\Gamma_{12}=\widetilde{\Gamma}\left(\xi_{2}, 2\right)$ и кусочногладкая функция $\lambda(\xi)$ удовлетворяет уравнению Уизема (1.6).

Величина $S\left(\lambda(\xi) \mid \lambda_{j}\right)$ обозначает так же, как и выше, групповую скорость в уравнении (1.6), вычисленную по формуле (2.2).

Предположим, что выполнено условие

$$
\lim _{\lambda(\xi) \rightarrow \lambda_{1}} \operatorname{Re} S\left(\lambda(\xi) \mid \lambda_{1}, \lambda(\xi)\right)<\lim _{\lambda(\xi) \rightarrow \lambda_{2}} \operatorname{Re} S\left(\lambda(\xi) \mid \lambda(\xi), \lambda_{2}\right) .
$$

Тогда справедлива следующая

ТеОрема 3.3. Для любых граничных условий рода 1

$$
\Gamma(\xi) \rightarrow \begin{cases}\Gamma_{1}, & \xi \rightarrow+\infty, \\ \Gamma_{2}, & \xi \rightarrow-\infty,\end{cases}
$$

удовлетворяющих условию (3.6), существует единственная кусочно-дладкал деформачия $\widetilde{\Gamma}(\xi)$ вида (3.4), (3.5), такал, что $\lambda(\xi)$ (локально) является решением уравнения Уизема (1.6).

ДокАЗАТЕльство. Выберем риманову поверхность $\widetilde{\Gamma}(\xi, 1)$ вида $(3.5)$ так, чтобы выполнялись соотношения

$$
\left\{\begin{array}{l}
\operatorname{Im} S\left(\lambda(\xi) \mid \lambda_{1}, \lambda(\xi)\right)=0 \\
\operatorname{Re} S\left(\lambda(\xi) \mid \lambda_{1}, \lambda(\xi)\right)=\xi
\end{array}\right.
$$

В силу леммы 3.1 кривая $\operatorname{Im} S=0$ проходит через точку $\lambda_{1}$ и пересекает вещественную ось в некоторой точке $\lambda\left(\xi_{\text {нач }}\right)=\bar{\lambda}\left(\xi_{\text {нач }}\right)$. В этой точке происходит «рождение» зоны $(\lambda(\xi), \bar{\lambda}(\xi))$.

По следствию теоремы 2.3 движение точки $\lambda(\xi)$ по кривой $\operatorname{Im} S=0$ происходит монотонно и определяется уравнением (3.2) $\operatorname{Re} S=\xi$. 


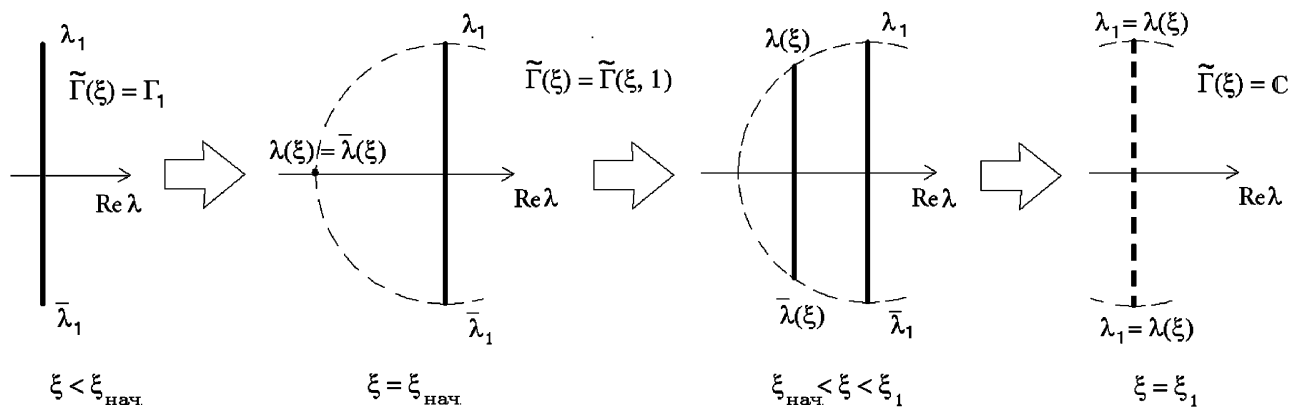

Рис. 2. Эволюция первой зоны $\left(\lambda_{1}, \bar{\lambda}_{1}\right)$.

В момент $\xi_{1}=\operatorname{Re} S\left(\lambda_{1} \mid \lambda_{1}, \lambda_{1}\right)$ произойдет «схлопывание» зон $(\lambda(\xi), \bar{\lambda}(\xi))$ и $\left(\lambda_{1}, \bar{\lambda}_{1}\right)$, что соответствует «нульзонной» поверхности $\widetilde{\Gamma}\left(\xi_{1}, 1\right)=\mathbb{C}$.

В силу условия $(3.6)$ нульзонная поверхность $\widetilde{\Gamma}\left(\xi_{1}, 1\right)=\mathbb{C}$ существует в интервале $\xi_{1}<\xi<\xi_{2}$, где $\xi_{2}=\operatorname{Re} S\left(\lambda_{2} \mid \lambda_{2}, \lambda_{2}\right)$. В момент $\xi_{2}$ происходит «рождение» двухзонной поверхности $(3.5) \widetilde{\Gamma}(\xi, 2)$, где $\lambda\left(\xi_{2}\right)=\lambda_{2}, \bar{\lambda}\left(\xi_{2}\right)=\bar{\lambda}_{2}$. По лемме 3.1 существует решение уравнения $\operatorname{Im} S\left(\lambda(\xi) \mid \lambda(\xi), \lambda_{2}\right)=0$, удовлетворяющее условию $\lambda\left(\xi_{2}\right)=\lambda_{2}$, а по теореме 3.1 и следствию теоремы 2.3 это

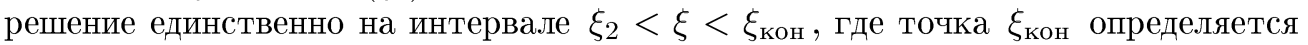
в силу леммы 3.3 из условия «схлопывания» зоны $(\lambda(\xi), \bar{\lambda}(\xi))$ на вещественной оси: $\xi_{\text {кон }}=S\left(\lambda\left(\xi_{\text {кон }}\right) \mid \lambda\left(\xi_{\text {кон }}\right), \lambda_{2}\right), \lambda\left(\xi_{\text {кон }}\right)=\bar{\lambda}\left(\xi_{\text {кон }}\right)$.
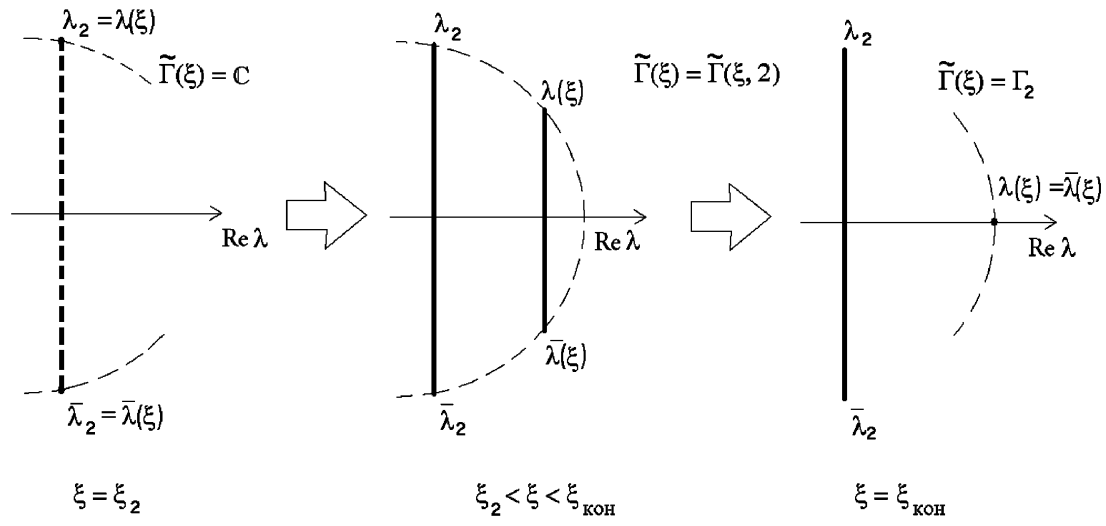

Рис. 3. Эволюция второй зоны $\left(\lambda_{2}, \bar{\lambda}_{2}\right)$.

В этот момент заканчивается эволюция римановой поверхности $\widetilde{\Gamma}(\xi)$, т.е. далее $\widetilde{\Gamma}(\xi)=\Gamma_{2}$ при $\xi>\xi_{\text {кон }}$.

Таким образом построена кусочно-гладкая поверхность (3.4), удовлетворяющая уравнению Уизема. Ее единственность вытекает из условия непрерывности уиземовской деформации и теорем 2.3 и 3.1. Теорема доказана. 
ЗАМЕЧАНИЕ 1. Обсудим подробнее гладкость решения исходного уравнения синус-Гордон в точках «схлопывания» или «рождения» зон. В общем случае предложенная в теореме 3.3 конструкция обеспечивает лишь непрерывность приближенного решения $u(x, t)$, восстановленного по формуле (1.2). Однако в ряде случаев из нее вытекает гладкость построенного решения. Так, например, в момент $\xi=\xi_{1}$, отвечающий вырождению двухзонной поверхности $\widetilde{\Gamma}(\xi)$ в нульзонную (см. рис. 2), решение (1.2) вырождается в бризер

$$
u(x, t) \rightarrow 4 \operatorname{arctg} \frac{\operatorname{tg} \varphi_{1} \cos \left(\left(t-\xi_{1} x\right)\left(1-\xi_{1}^{2}\right)^{-1} \cos \varphi_{1}\right)}{\operatorname{ch}\left(\left(t-\xi_{1}\right)\left(1-\xi_{1}^{2}\right)^{-1} \sin \varphi_{1}\right)}, \quad x \rightarrow \xi_{1} t,
$$

где $\lambda_{1}=\left|\lambda_{1}\right| e^{2 i \varphi_{1}}, \xi_{1}=\left(1-16\left|\lambda_{1}\right|\right) /\left(1+16\left|\lambda_{1}\right|\right)$.

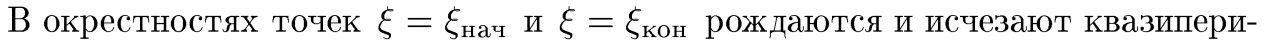
одические волны малой амплитуды, и вопрос об их гладкой склейке с заданными периодическими граничными решениями требует отдельного рассмотрения. Отметим лишь, что подобный анализ проделывался в задаче Гуревича-Питаевского

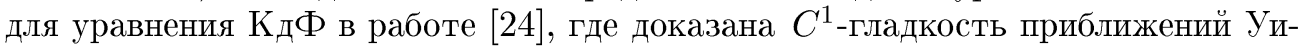
зема в переходных областях.

ЗАмЕчАниЕ 2. Обобщение теоремы 3.3 на случай граничных условий (3.7) произвольного рода представляется существенно более трудной задачей. Ясно, что комплексные кривые $\operatorname{Im} S=0$ имеют даже в случае рода 3 значительно более сложную структуру, а поскольку в общем случае отсутствует какая-либо монотонность $\operatorname{Re} S$ по $\lambda_{j}$, приходится налагать условия вида (3.6) для каждой пары точек $\lambda_{i}$ и $\lambda_{j}$, соединяемых деформацией $\lambda(\xi)$ в силу уравнений (3.8). В свою очередь, подобные дополнительные условия могут привести к нарушению единственности решения, поскольку в случае рода $N>2$ возможны различные комбинации сценариев «рождения» и «схлопывания» зон типа изображенных на рис. 2,3 .

\section{§4. Приложение. Вычисление определителей}

Напомним, что в основном тексте использовалось символическое обозначение определителя (2.1), где интегралы по циклам охватывают точки ветвления $\lambda_{i}$, $\bar{\lambda}_{i}$, как показано на рис. 1.

ПреДЛОЖЕНИЕ 1. При любых $\lambda_{1}, \ldots, \lambda_{N}$ определитель $\left|1, \mu, \ldots, \mu^{N-1}\right|$ отличен от нуля.

ДокАЗАТЕЛЬСтво. Пусть, напротив, $\left|1, \mu, \ldots, \mu^{N-1}\right|=0$. Тогда в силу линейной зависимости его столбцов найдется многочлен $P_{N-1}$ степени $N-1$, такой, что голоморфный абелев дифференциал $P_{N-1}(\mu) R^{-1}(\mu) d \mu$ имеет нулевые $a$-периоды, что невозможно (см. $[12$, с. 285]).

ПРЕДЛОЖЕНИЕ 2. Пусть $\lambda_{1}-$ точка ветвления, такая, что $\operatorname{Im} S\left(\lambda_{1}\right)$ $=0$. Тогда для любой точки $\lambda$, такой, что $\lambda \neq \lambda_{1}, \lambda \neq \bar{\lambda}_{1}$, определитель $\Delta(\Lambda)=\left|\Lambda \mu^{-1}, \Lambda, \ldots, \Lambda \mu^{N-2}\right|$ отличен от нуля при $\Lambda=\left(\lambda_{1}-\mu\right)(\lambda-\mu)$.

ДокаЗАТЕЛЬСТво. Если $\operatorname{Im} S\left(\lambda_{1}\right)=0$, то по теореме 2.2 имеем $\Delta\left(\left(\lambda_{1}-\mu\right)\left(\bar{\lambda}_{1}-\mu\right)\right)=0$. Предполагая от противного, что $\Delta(\Lambda)=0$, и выражая из этого равенства абелевы интегралы с особенностью в нуле через голоморфный базис $\mu^{k} R^{-1}(\mu) d \mu$, получим голоморфный дифференциал с нулевыми 
$a$-периодами. Непосредственный счет показывает, что его коэффициенты равны нулю только при $\lambda=\lambda_{1}$ или $\lambda=\bar{\lambda}_{1}$, что и доказывает предложение.

СлЕдСТВИЕ. При $\operatorname{Im} S\left(\lambda_{j}\right)=0$ в точках ветвления $\Delta\left(\left(\lambda_{j}-\mu\right)^{2}\right) \neq 0$.

ПРЕДЛОЖЕНИЕ 3. Пусть $N=2 ; \operatorname{mozдa~} \Delta=\Delta\left(\left(\lambda_{j}-\mu\right)\left(\bar{\lambda}_{j}-\mu\right)\right), j=1,2$, равны нулю при $\left|\lambda_{1}\right|=\left|\lambda_{2}\right|$.

ДокАЗАТЕльство. Положим $\lambda_{1}=r e^{i \phi_{1}}, \lambda_{2}=r e^{i \phi_{2}}$ и перейдем в гиперэллиптических интегралах к переменной $\phi: \mu=r e^{i \phi}$. Тогда

$$
\begin{gathered}
\Delta_{k 1}=\sqrt{r} \int_{-\phi_{j}}^{\phi_{j}} e^{i \phi / 2} F(\phi) d \phi, \quad \Delta_{k 2}=\frac{1}{\sqrt{r}} \int_{-\phi_{j}}^{\phi_{j}} e^{-i \phi / 2} F(\phi) d \phi, \\
F(\phi)=\left(\frac{\left(e^{i \phi}-e^{i \phi_{1}}\right)\left(e^{i \phi}-e^{-i \phi_{1}}\right)}{\left(e^{i \phi}-e^{i \phi_{2}}\right)\left(e^{i \phi}-e^{-i \phi_{2}}\right)}\right)^{1 / 2} .
\end{gathered}
$$

В интегралах (П1) мы преобразовали контуры $a_{1}$ и $a_{2}$ к дугам окружности $|\mu|=r$, воспользовавшись инвариантностью определителя $\Delta$ при замене $a_{2} \mapsto$ $a_{2} \pm a_{1}$ во второй строке. Непосредственная проверка демонстрирует четность и вещественность функции (П2): $F(-\phi)=F(\phi)=\bar{F}(\phi)$. Выполняя замену $\phi \mapsto-\phi$ в интегралах (П1), получаем пропорциональность строк определителя, что и доказывает предложение.

\section{ЛИТЕРАТУРА}

1. Захаров В. Е, Манаков С. В., Новиков С. П., Питаевский Л. П. Теория солитонов. Наука, M., 1980.

2. Forest M. G., McLaughlin D. V. Modulation of sinh-Gordon and sine-Gordon wavetrains. Stud. Appl. Math., 68, 11-60 (1983).

3. Bikbaev R. F., Novokshenov V. Yu. Self-similar solutions of the Whitham equations and Korteweg-de Vries equations with finite-gap boundary conditions. In: Proc. III Intern. Workshop "Nonlinear and turbulent processes in physics", Vol. 1, Kiev, 1986, pp. 32-35.

4. Бикбаев Р. Ф., Новокшенов В. Ю. Уравнение КдФ с конечнозонными граничными условиями и однопараметрические решения уравнений Уизема. В сб.: «Асимптотические свойства решений дифференциальных уравнений», Уфа, 1989 , с. 3-35.

5. Бикбаев P. Ф., Шарипов P. A. Асимптотика решения задачи Коши для уравнения Кортевега-де Фриза в классе потенциалов с конечнозонным поведением при $t \rightarrow \infty$. Теорет. матем. физ., 78, 345-356 (1989).

6. Levermore D. Hyperbolic structure of the $\mathrm{KdV}$ zero dispersion limit. Commun. Partial Diff. Equat., 13, 485-511 (1988)

7. Бикбаев P. Ф. Перестройка Уизема и временна́я асимптотика решения нелинейного уравнения Шрёдингера с конечнозонным поведением при $x \rightarrow \pm \infty$. Алгебра и анализ, 2, 131-143 (1990).

8. Бикбаев Р. Ф., Куксин С. Б. Периодическая граничная задача для уравнения синус-Гордон, его малые гамильтоновы возмущения и КАМ-теория для конечнозонных торов. Алгебра и анализ, 4, 42-78 (1992).

9. Date E. Multi-soliton and quasi-periodic solution of nonlinear equations of sineGordon type. Osaka J. Math., 19, 125-158 (1982).

10. Erkolani N., Forest M. G. On the manifold of real quasi-periodic sine-Gordon solutions. Commun. Math. Phys., 99, 3-48 (1985). 
11. Дубровин Б. А., Натанзон С. М. Вещественные двухзонные решения уравнения sine-Gordon. Функц. анализ и его прил., 16, 27-43 (1982).

12. Спрингер Дж. Римановы поверхности. Мир, М., 1975.

13. Новокшенов В. Ю. Уиземовские деформации интегрируемых систем типа волчков. Функц. анализ и его прил., 52, 50-62 (1993).

14. Дубровин Б. А., Новиков С. П. Гидродинамика слабо деформированных солитонных решеток. Дифференциальная геометрия и гамильтонова теория. УМН, 44, 29-98 (1989).

15. Кричевер И. М. Метод усреднения для двумерных интегрируемых уравнений. Функц. анализ и его прил., 22, 37-52 (1988).

16. Камчатнов A. М. Нелинейные периодические волны в вынужденном комбинационном рассеянии света и рождение солитонов на фронте импульса. ЖЭТФ, 109, 786-804 (1996).

17. Bikbaev R. F., Kudashev V. R. Example of shock waves in unstable media: the focusing nonlinear Schrödinger equation. Phys. Lett. A, 190, 255-258 (1994).

18. Гуревич А. В., Крылов А. Л., Эль Г. А. Эволюция римановой волны в дисперсионной гидродинамике. ЖЭЭФ, 101, 1797-1807 (1992).

19. El' G. A., Gurevich A. V., Khodorovskii V. V., Krylov A. L. Modulation instability and formation of a nonlinear oscillatory structure in a "focusing" medium. Phys. Lett. A, 177, 357-361 (1992).

20. Krichever $I$. $M$. The periodic problem for two-dimensional integrable system. In: Proc. Int. Congress of Math., Springer, Kyoto, 1991, pp. 1353-1362.

21. Krichever I. M. The dispersionless Lax equations and topological minimal models. Commun. Math. Phys., 143, 415-429 (1992).

22. Forest $M$. G., Sinha A. A numerical study of nearly integrable modulation equations. In: Singular Limits of Dispersive Waves, eds. N. Ercolani et al., Plenum, New York, 1994, pp. 39-52.

23. Bronski J. C., McLaughlin D. W. Semiclassical behavior in the NLS equation, optical shocks-focusing instabilities. In: Singular Limits of Dispersive Waves, eds. N. Ercolani et al., Plenum, New York, 1994, pp. 12-25.

24. Потемин Г. В. Алгеброгеометрические конструкции автомодельных решений уравнений Уизема. УМН, 43, вып. 5, 252-253 (1988). 